\title{
Strain and Spin-Orbit Effects in Self-Assembled Quantum Dots
}

\author{
M. Zieliński ${ }^{a}$, W. JASKÓLSKI ${ }^{a}$, J. AizPURUA ${ }^{b}$ \\ AND G.W. BRYANT ${ }^{c}$ \\ ${ }^{a}$ Instytut Fizyki UMK, Grudziądzka 5, 87-100 Toruń, Poland \\ ${ }^{b}$ Donostia International Physics Center, 20018 San Sebastian, Spain \\ ${ }^{c}$ National Institute of Standards and Technology \\ Gaithersburg, MD 20899-8423, USA
}

The effects of strain and spin-orbit interaction in self-assembled lens-shaped InAs/GaAs quantum dots are investigated. Calculations are performed with empirical tight-binding theory supplemented by the valence force field method to account for effects of strain caused by lattice mismatch at the InAs-GaAs interface. It is shown that both effects influence strongly the electron and hole energy structure: splitting of the energy levels, the number of bound states, density distributions, and transition rates. We show that piezoelectric effects are almost negligible in quantum dots of the size investigated.

PACS numbers: $71.35 . \mathrm{Cc}$

\section{Introduction}

Self-assembled InAs/GaAs nanoheterostructures receive much attention due to their potential applications as efficient quantum dot lasers $[1,2]$ or qubits in future quantum computers $[3,4]$. Such applications will require technological breakthroughs in the fabrication of uniform and repeatable quantum dots (QD). There is also still a need for precise theoretical descriptions of the electronic and optical properties of such systems. The growth of self-assembled quantum dots is driven by the effects of strain caused by lattice mismatch at the interfaces separating different semiconductor components. Strain is therefore an important factor determining the energy structure and optical properties of self-assembled quantum dots $[2,5,6]$. Piezoelectric effects have also been shown to be important, at least in large enough quantum dots $[7,8]$. Recently, it has been shown that atomistic models, like the pseudopotential [8] and empirical tight-binding approaches (ETB), are the most adequate to properly account for these effects. These methods are able to account for atomistic effects that are missed by continuous medium approaches. 
Relativistic effects are also known to be large in both InAs and GaAs $[9,10]$ and therefore spin-orbit effects must be taken into account to properly describe the valence-band (VB) states of InAs/GaAs quantum dots. In this paper we study the contribution of strain, spin-orbit and piezoelectric effects to the energy structure and transition rates in lens-shape quantum dots. Working in the framework of the empirical tight-binding approach [11] we calculate one-particle electron and hole energy levels and transition rates. We study how the electronic structure and optical spectra of such dots depend on the different effects included in the theory. We show that including these effects significantly affects the number of bound states, changes density distributions and level splittings, and alters the optical spectra.

\section{Theory}

\subsection{System and method description}

The InAs quantum dot we investigate are lens-shaped with a height $h=$ $1.8 \mathrm{~nm}$ and a base diameter $d=7 \mathrm{~nm}$. These values correspond to the smallest sizes of quantum dots attainable in the Stransky-Krastanov mode growth* $[12,13]$. The lens is situated on a 2 monolayer (ML) thick wetting layer (WL) and is embedded in a GaAs buffer. We have chosen a cylindrical shape for the finite external GaAs buffer that surrounds the dot in order to not perturb the internal symmetry of the QD states. For both the lattice relaxation and the tight-binding calculations the surrounding GaAs buffer has to be large enough to ensure that further size increase will not change significantly the strain field around the quantum dot or the bound state energies and the level ordering in the quantum dot.

Initially the atoms occupy the sites of a uniform zinc-blende lattice. In our TB approach each atom is described by the $s, p_{x}, p_{y}, p_{z}$, and $s^{*}$ orbitals [11]. Only coupling of on-site and nearest neighbor orbitals is included. This results in 13 TB empirical parameters, which for the unstrained system are obtained by fitting the bulk TB results to experimentally known band gaps and effective masses [14]. Surface states are excluded by passivating the surface dangling bonds. This is done by shifting their energies high above the conduction band (CB) so that they do not modify states near the band gap $[11,15,16]$. The valence band offset between InAs and GaAs is taken as $0.2 \mathrm{eV}$. Finding TB states before performing lattice relaxation corresponds to the approximation in which the effects of strain are neglected.

\subsection{Strain effects}

The initial uniform lattice of the InAs QD and GaAs buffer has the zinc-blende structure with the GaAs lattice constant. This is because we assume that far away from the InAs quantum dot the GaAs buffer remains unstrained. Such a

\footnotetext{
${ }^{*}$ Unlike the dots studied in Ref. [8], they have the height-to-base ratio closer to experimentally grown lens-shaped dots.
} 
uniform system exhibits enormous strain, since all InAs nodes are far from their bulk positions. To minimize the strain energy we perform lattice relaxation using the valence force field (VFF) method $[17,18]$. We assume the following boundary conditions (BC) for the lattice relaxation: atoms at the bottom and top surfaces of the cylindric GaAs buffer can move freely in any direction, while the positions of atoms at the lateral surface of the cylinder are fixed. Such boundary conditions allow us to relax strain in the $z$-direction away from the quantum dot, but to prevent leakage (expansion) of the InAs WL out of the GaAs cylinder.

The minimization of the strain energy (lattice relaxation) is performed using a combination of the steepest descent and conjugate gradient techniques [19]. The minimization process stops when the maximum force applied to each atom is less than $6 \times 10^{-6} \mathrm{eV} / \mathrm{nm}$. With the lattice relaxed and all the atoms in their new positions, the local strain tensor and strain profiles are calculated [17] and visualized. These values help to understand how the strain effects influence polarization and splitting of the one-particle states in quantum dots. We have found that to get values of trace of the strain tensor, $\operatorname{Tr}(\epsilon) \approx 0.001$ at the GaAs buffer surface (i.e., 100 times less than the final values of $\operatorname{Tr}(\epsilon)$ at the relaxed InAs/GaAs interface), one has to consider GaAs buffer of diameter equal to $38 \mathrm{~nm}$ and height $33 \mathrm{~nm}$. Such a huge buffer contains almost $1.5 \times 10^{6}$ atoms.

For the new atomic positions and thus new bond lengths and bond angles, the TB off-site parameters, $H_{i j}, i \neq j$, are recalculated for each pair of neighboring atoms. We use the Slater-Koster formulas [20] to incorporate the effect of changed bond angles on $H_{i j}$ and power-law scaling for the bond lengths $H_{i j}=H_{i j}^{0}\left(d_{i j}^{0} / d_{i j}\right)^{\kappa}$, where $d_{i j}$ are the bond lengths and subscript 0 corresponds to the unstrained values. The exponent $\kappa$ is determined by fitting the InAs and GaAs volume deformation potentials under hydrostatic pressure to their experimental values. The details are given in Ref. [5]. The resulting exponent is 2.9. The TB parameters can also change if piezoelectric potential, which results from the strained (distorted) lattice, is taken into account. This change corresponds to the addition of the piezoelectric potential to the on-site diagonal terms $H_{i i}$. The calculation of piezoelectric charge follows the procedure described in Ref. [18], while the piezoelectric potential is calculated by numerically solving the Poisson equation on a cubic grid.

Finally, the TB Hamiltonian matrix is constructed and the required one-particle states are found. Before we diagonalize the TB Hamiltonian, we cut the GaAs buffer to a size that makes this diagonalization more feasible. The diameter of the buffer (from now on called the TB buffer) is $15.3 \mathrm{~nm}$ and its height is $10.6 \mathrm{~nm}$. The TB buffer contains 77551 atoms. Increasing the size of the buffer changes the ground electron and hole energy levels of the order of $1 \mathrm{meV}$.

\subsection{Spin-orbit effects}

The extension of the one-particle Hamiltonian, $H$, to include the relativistic effects is usually done via the Pauli corrections. We assume that spin-orbit (SO) 
interaction, $H^{\text {so }}$, mixes only on-site $p$-type spin-up and spin-down orbitals. It introduces two additional TB parameters, one for anions and one for cations ${ }^{\dagger}$. Since now each atomic orbital is associated with a spin state, the dimension of the Hamiltonian matrix doubles and each on-site Hamiltonian sub-block becomes non-diagonal and complex. For systems containing more than $10^{5}$ atoms, this substantially slows the process of finding the TB states, that, additionally, are doubly degenerate. One can reduce the numerical effort and estimate the spinorbit effects by performing calculations using a limited basis of the one-particle TB states found without SO interactions.

Comparison of the results obtained with and without inclusion of the spinorbit effects is not straightforward, because different sets of the TB parameters have been used in the literature to reproduce the same bulk energy gaps and effective masses. We use two sets of bulk TB parameters: one without SO (13 parameters) from Vogl et al. [14] (VHD) and the other with SO (15 parameters) from Klimeck et al. [21] (KBC). Both sets of parameters yield approximately correct bulk gaps and effective masses, but the VHD parameters do not account for the SO-induced valence band splittings. However, since the VHD parameters are found by fitting to experimental energy gaps and effective masses, they include SO effects indirectly.

In contrast, calculations performed using the $\mathrm{KBC}$ parameters with the $\mathrm{SO}$ parameters set to zero (KBC-NSO (no spin-orbit) calculations) yield incorrect bulk gaps and effective masses. We have checked that a treatment of $H^{\text {so }}$ using a limit basis of 30 hole and 30 electron functions found from the KBC-NSO calculations, do yield QD bound states energies close to the energies obtained with the full KBC set including SO effects (KBC-SO energies). Calculations performed with a limited basis of functions originating from the VHD calculations and with spin-orbit parameters taken from the KBC set, do not converge to the KBC-SO energies.

\subsection{Transition rates}

The single-particle electron-hole transition rates are calculated by evaluating the dipole matrix elements in real space using the TB wave functions. These matrix elements are expressed in terms of dipole moments for the basis orbitals on the same atom and for the overlapping $s p^{3}$ hybridized bond-orbitals from nearest neighbors. The on-site dipole matrix elements are approximated by the values of atomic dipole moments given in Ref. [22]. The dipole moments for nearest neighbors are chosen by reasonable estimates. The details are given in Ref. [11]. The choices made for bond-orbital dipole matrix elements are not critical. The symmetries of the electron and hole states determine which electron-hole transitions are optically active.

${ }^{\dagger}$ We assume that $H^{\text {so }}$ couples only the on-site orbitals. 


\section{Results}

\subsection{Strain fields}

The zinc-blende lattice reduces the cylindrical symmetry of lens-shaped objects to $C_{2 v}$ symmetry with different arrangement of atoms at InAs/GaAs interfaces when moving in [110] and [110] directions. This effect is called the atomistic interface effect [8]. In the TB theory (as well as in any atomistic approach) it manifests itself in a small splitting of the $p$-type electron levels (usually $e_{2}$ and $e_{3}$, see next subsection). Strain enhances this effect, since strain causes lattice distortion at the interface. Analysis of the strain tensor allows us to study such effects and helps to understand the TB results obtained after lattice relaxation in comparison to the approximation which neglects strain. Figure 1a shows the difference of the
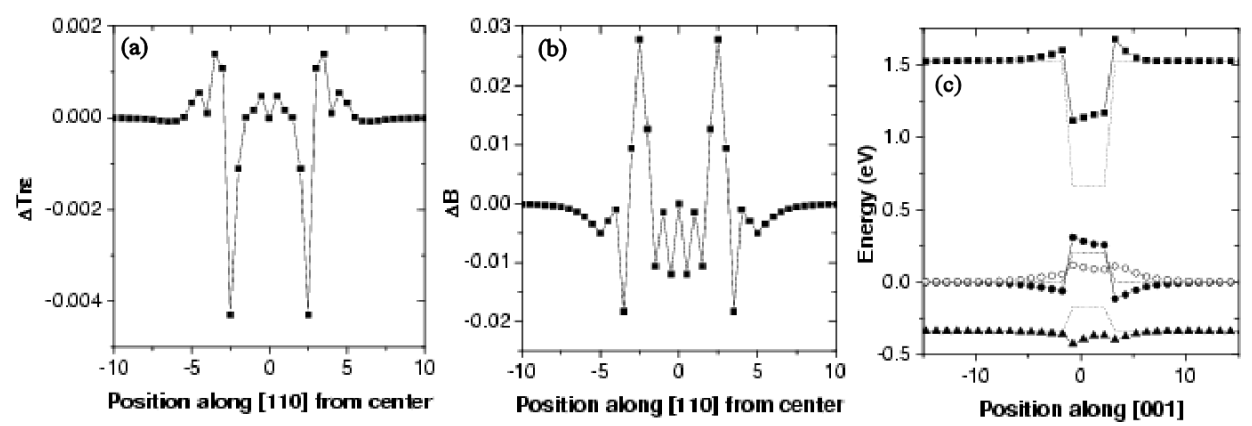

Fig. 1. Differences of $\operatorname{Tr}(\epsilon)$ (a) and biaxial strain component $B$ (b) in [110] and [1 10$]$ directions for relaxed QD vs. cation position. The values have been taken at $h=1.16 \mathrm{~nm}$ above the QD base center. Strain-induced confining potentials (c) along [001]. CB squares, heavy hole - closed circles, light hole — open circles, split-off band - triangles. Bulk unstrained GaAs/InAs band off-sets — dotted line.

$\operatorname{Tr}(\epsilon)$ (hydrostatic strain) for the [110] and [110] directions, which is responsible for increased splitting of the electron $p$-type states. Figure $1 \mathrm{~b}$ shows also the biaxial component of strain, i.e., $B=\left[\left(\epsilon_{x x}-\epsilon_{y y}\right)^{2}+\left(\epsilon_{y y}-\epsilon_{z z}\right)^{2}+\left(\epsilon_{z z}-\epsilon_{x x}\right)^{2}\right]$, which is responsible for splittings and polarizations of the hole states due to the strain effects.

Although TB calculations directly include the strain effects (via modification of the off-site parameters), the strain tensor elements and deformation potentials [17] can be used to estimate strain-induced confinement potentials for the electrons and holes. In Fig. 1c the conduction, heavy hole, light hole and split-off band profiles along [001] direction are shown and are compared to the bulk unstrained GaAs/InAs band off-sets. One can observe that the QD electron well gets significantly shallower, which leads to reduction of the number of bound states. Biaxial strain modifies the hole wells in a very different way, thus leading to complex mixing of the valence subbands. 


\subsection{Electronic structure}

We have performed calculations in several approximations: (i) without strain and without spin-orbit effects (NSTR-NSO), (ii) with strain effects included, but without spin-orbit interaction (STR-NSO), (iii) without strain effects, but including spin-orbit effects (NSTR-SO), (iv) with both the strain and spin-orbit effects taken into account (STR-SO). Piezoelectric effects have been added to (ii) and (iv); the corresponding results are denoted as STR-NSO-P and STR-SO-P, respectively.

Let us consider first an InAs wetting layer without a QD. In this case, the ground electron and hole energy levels mark the thresholds for the conduction and valence band continua of the WL. These thresholds change when we go from NSTR to STR approximations. They are schematically shown in Fig. 2. When the effects of strain are taken into account, the energy of the lowest WL electron level increases by $116 \mathrm{meV}$, while the top of the WL hole continuum increases by only $9 \mathrm{meV}$; the gap increases by $\approx 107 \mathrm{meV}$. Since the InAs lattice constant $a_{\text {InAs }}$ is greater than $a_{\mathrm{GaAs}}$, the InAs wetting layer undergoes biaxial compressive strain. The shifts of the energy thresholds observed in Fig. 2 (when going from NSTR to STR approximations) agree well with the shifts predicted in Ref. [23] for the CB and VB edges of biaxially compressed quantum wells.

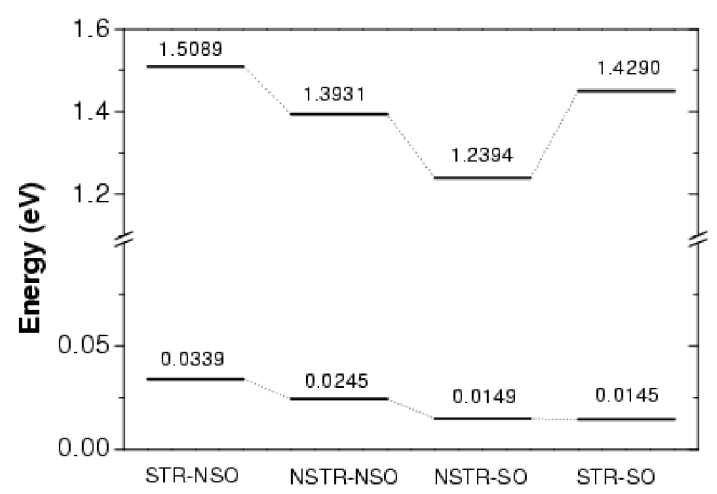

Fig. 2. InAs wetting layer conduction band and valence band edges (eV) calculated in different approximations.

In comparison to the NSTR-NSO approximation, the spin-orbit interaction lowers the InAs WL CB edge by $154 \mathrm{meV}$ and the VB edge by $10 \mathrm{meV}$; the gap decreases by $\approx 144 \mathrm{meV}$. For the approximations that count for the strain effects, these values are $80 \mathrm{meV}$ and $19 \mathrm{meV}$, respectively and the gap decreases by $\approx 60 \mathrm{meV}$. The effect of spin-orbit interaction is therefore opposite to the effect of strain ${ }^{\ddagger}$. Figure 2 shows also that the influence of these effects on the WL band edges is not additive.

\footnotetext{
${ }_{\ddagger}^{\ddagger}$ The decrease in the energy gap after inclusion of the spin-orbit interaction may to some extent be caused also by different description of the bulk properties by the VHD and $\mathrm{KBC}$ parameterizations.
} 
Let us consider now the quantum dots states. To recognize whether a given one particle state is bound and localized in the quantum dot, one has to check its energy versus the corresponding WL threshold and analyze its density distribution. If the energy of a given QD electron state is well above (well below for the hole states) the corresponding threshold, the state is classified as the unbound WL state. The QD states which have energies close to the thresholds are classified as bound if their densities do not leak to the very edges of the WL.

The energies of the three lowest electron states and three highest hole states calculated in the approximations (i)-(iv) are presented in Table. The corresponding densities are visualized in Figs. 3 and 4 . Table shows also the number of

TABLE

Energies $(\mathrm{eV})$ of the bound electron and hole states of InAs/GaAs lens-type QD (measured from the bulk GaAs VB-edge), calculated in different approximations (see text). The effective gap $E_{\mathrm{g}}$ and the number of electron $\left(e_{\mathrm{b}}\right)$ and hole $\left(h_{\mathrm{b}}\right)$ bound states are also shown.

\begin{tabular}{c|c|c|c|c|c|c}
\hline \hline & NSTR-NSO & NSTR-SO & STR-NSO & STR-NSO-P & STR-SO & STR-SO-P \\
\hline$e_{3}$ & 1.2728 & 1.2135 & 1.5095 & 1.5097 & 1.4450 & 1.4453 \\
$e_{2}$ & 1.2715 & 1.2111 & 1.4929 & 1.4904 & 1.4140 & 1.4113 \\
$e_{1}$ & 1.1218 & 1.0309 & 1.4032 & 1.4016 & 1.2971 & 1.2953 \\
\hline$h_{1}$ & 0.1021 & 0.0774 & 0.0741 & 0.0684 & 0.0728 & 0.0701 \\
$h_{2}$ & 0.0992 & 0.0655 & 0.0699 & 0.0650 & 0.0660 & 0.0637 \\
$h_{3}$ & 0.0830 & 0.0554 & 0.0567 & 0.0550 & 0.0445 & 0.0423 \\
\hline$E_{\mathrm{g}}$ & 1.0198 & 0.9535 & 1.3290 & 1.3332 & 1.2243 & 1.2252 \\
\hline$e_{\mathrm{b}}$ & 6 & 3 & 3 & 3 & 2 & 2 \\
$h_{\mathrm{b}}$ & 17 & 10 & 7 & 7 & 8 & 8
\end{tabular}

electron and hole bound states found in each case. The strain not only raises the energy of the ground QD electron state $e_{1}$, as it increases the WL CB continuum threshold, but it also makes this state less bound. When the strain effects are added to the NSTR-NSO approximation the binding energy of $e_{1}$ state decreases by about $166 \mathrm{meV}$. Similarly, when strain is added to the calculations that already count for the spin-orbit interaction, the binding energy of $e_{1}$ decreases by $76 \mathrm{meV}$. The weaker binding of the QD electron states manifests itself by more defused densities (see Fig. 3); the third electron state in the STR-SO case is so defused that it should already be classified as the unbound state. Additionally, lattice relaxation enhances splitting between the $p$-type $\left(e_{2}\right.$ and $\left.e_{3}\right)$ levels from $1.5 \mathrm{meV}$ (the so-called atomistic interface effect) to $16 \mathrm{meV}$, in agreement with recent results of Ref. [8] obtained in the pseudopotential approach. The weaker (shallower) QD electron well of the relaxed lattice leads also to a decrease in the separation energy between the ground $e_{1}$ and the first excited $e_{2}$ state. 


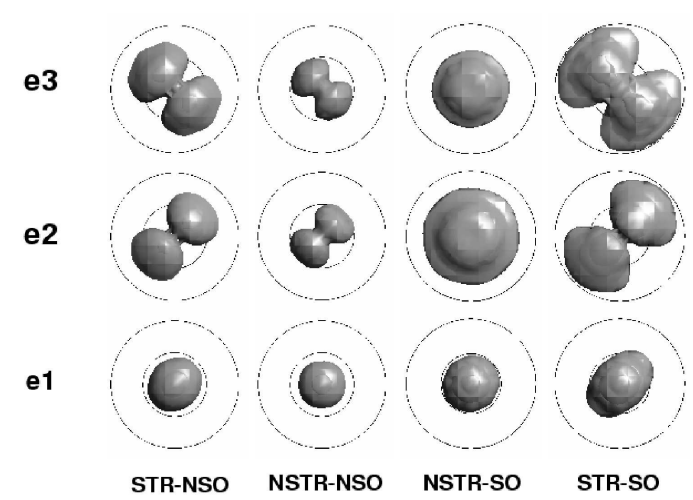

Fig. 3. Density isosurfaces (50\%) of three bound electron states of InAs QD calculated in different approximations. Inner circle marks the base of QD; outer circle marks the size of GaAs TB buffer.

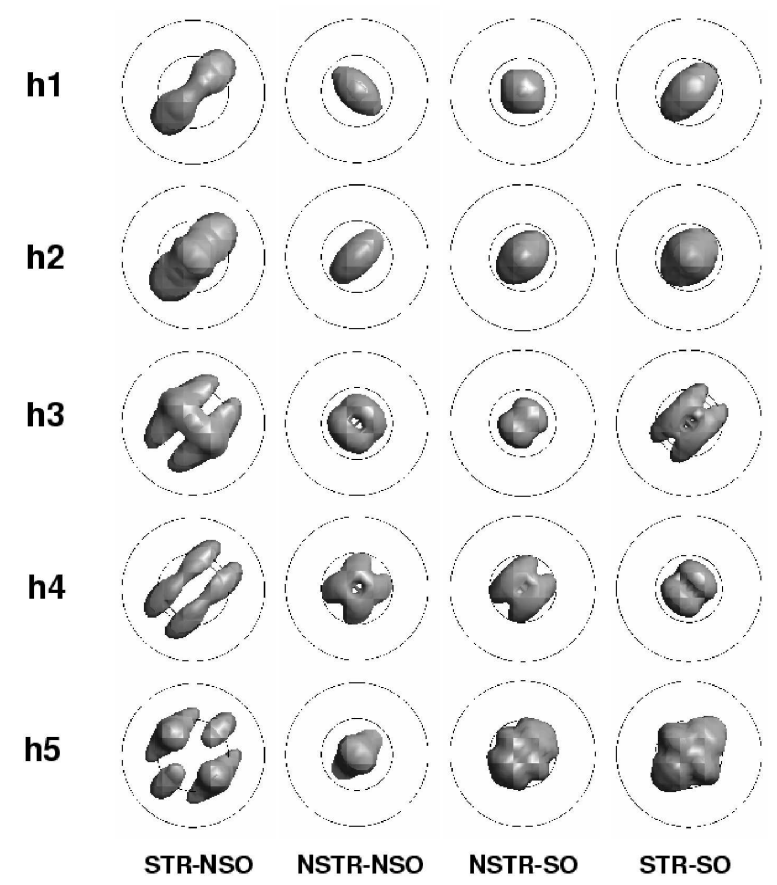

Fig. 4. Density isosurfaces (50\%) of five bound hole states of InAs QD calculated in different approximations.

Let us consider now the energies and density distributions of the bound electron states after the inclusion of spin-orbit interaction (in comparison to the NSTR-NSO approximation). Although the binding energy of $e_{1}$ state does not change, the number of bound states is reduced from 6 to 3 . This is mainly due 
to the increase in the level splitting, due to the differences in $\mathrm{CB}$ curvatures for the VHD and KBC parameterizations. SO calculations performed with a limited basis of 20 electron and 20 hole NSO functions (obtained by neglecting spin-orbit parameters in the KBC set) show that the spin-orbit effect on the energies of bound electron states is less than $5 \mathrm{meV}$. The NSTR-SO approximation leads to strong depolarization of $e_{2}$ and $e_{3}$ states $^{\S}$ (Fig. 3). Before adding spin-orbit interaction, the splitting and polarization of these states was due to weak atomic interface effect. The observed depolarization seems to be a "pure" spin-orbit effect. This is confirmed by Fig. 5, which shows densities of the bound electron states calculated in the NSTR-NSO approximation with the KBC parameters and by a limited basis treatment of the spin-orbit interaction. Finally, the inclusion of both the strain effects and spin-orbit interaction restores polarization of the $e_{2}$ and $e_{3}$ levels and enhances their splitting up to $30 \mathrm{meV}$.

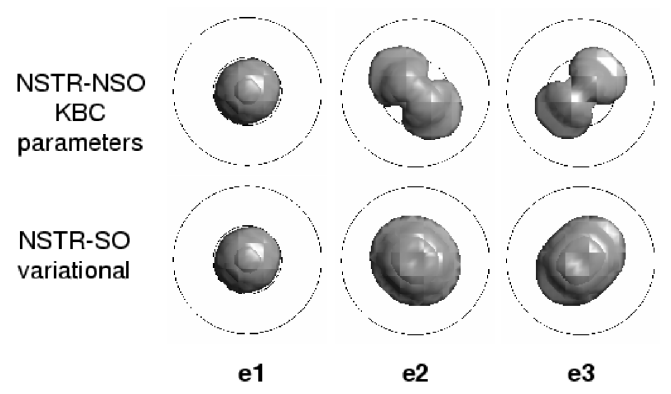

Fig. 5. Density isosurfaces (50\%) of three bound electron states calculated using only non-spin-orbit KBC parameters (top) and treating spin-orbit interaction variationally (bottom).

The influence of the strain effects and spin-orbit interaction on the hole states is more complex. Densities of the first five hole band states, calculated in different approximations are shown in Fig. 4. Lattice relaxation (compared to the NSTR-NSO approximation) reduces the number of the bound hole energy levels from 17 to 7 . Strain effects elongate all the bound hole states densities along the [110] direction. This is because the hole states are affected not only by the hydrostatic strain, as happens for the electron states, but also by the biaxial strain [8] that differs in the [110] and [110] directions (see Fig. 1). The effect is so strong that it even changes the polarization of the ground hole state. On the other hand, addition of the spin-orbit interaction to the NSTR-NSO case also leads to a strong modification of the hole energy spectrum (see Table and Fig. 4). It is interesting that further addition of the strain effects yields only slight modifications of the hole spectrum (elongation of densities in the [110] direction and reordering

\footnotetext{
\$Since all the energy levels calculated in approximations (iii) and (iv) are doubly degenerate, the visualized densities concern the wave functions of the maximum spin-up projection.
} 
of $h_{3}$ and $h_{4}$ levels). This means that spin-orbit interaction enhances localization of the hole states in the internal part of QD and makes these states immune to the strain effects that dominate at the InAs/GaAs interfaces of the QD and WL.

The piezoelectric effects added to the STR-NSO approximation influence the electronic structure of the investigated QD in a negligible way (see Table). The energy of the ground electron state $e_{1}$ decreases by $1.6 \mathrm{meV}$. The energy of the ground hole state $h_{1}$ decreases by $5.7 \mathrm{meV}$. The energies of the second excited electron and hole states change by only $0.2 \mathrm{meV}$ and $1.7 \mathrm{meV}$, respectively. The splitting between the $p$-type $e_{2}$ and $e_{3}$ electron energy levels increase by $2 \mathrm{meV}$ (from $17 \mathrm{meV}$ to $19 \mathrm{meV}$ ). In the approximation that counts also for the spin-orbit interaction, the addition of the piezoelectric effects makes even smaller changes to the energy spectrum: the ground electron level decreases by $1.8 \mathrm{meV}$ and the $e_{2}, e_{3}$ splitting increases by $3 \mathrm{meV}$ (in comparison to STR-SO approximation). We do not observe any polarization exchange between $e_{2}$ and $e_{3}$ as reported in Ref. [8]. This is because our QD is smaller than the dots studied in the quoted work, where it was found that the influence of piezoelectric effects on the splitting of $e_{2}$ and $e_{3}$ states increases with the dot size.

\subsection{Transition rates}

Several lowest electron-hole transition rates for unpolarized light are presented in Fig. 6. Only the NSTR-NSO and STR-SO approximations are compared. Although the strain and spin-orbit effects lead to a strong blueshift $(\approx 200 \mathrm{meV})$ of the entire spectrum, the first three brightest lines correspond to the same three transitions: $e_{1}-h_{1}, e_{1}-h_{2}$ and $e_{1}-h_{4}$.

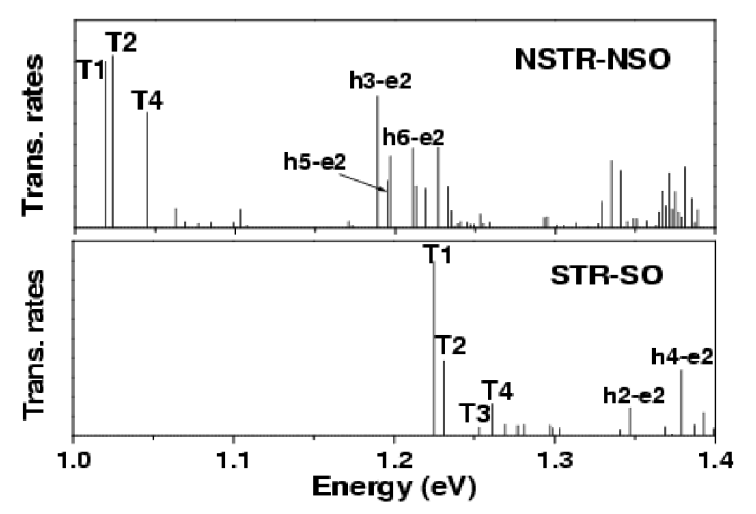

Fig. 6. Electron-hole transition rates (arbitrary units) of the InAs QD calculated in NSTR-NSO and STR-SO approximations. T1, T2, T3, and T4 mark transitions from $e_{1}$ electron state to $h_{1}, h_{2}, h_{3}$, and $h_{4}$ hole states, respectively.

They are marked as T1, T2 and T4. The $e_{1}-h_{3}$ transition is strongly forbidden in the NSTR-NSO approximation, since the $h_{3}$ wave function has a nodal surface in the [110] direction. This transition is weak, but not forbidden in the 
STR-SO case. This is because in the STR-SO approximation the spin-orbit interaction mixes the valence band states and the $h_{3}$ state has no sharply defined nodal surface.

\section{Conclusions}

We have investigated the effects of strain, spin-orbit interaction and piezoelectric potential on one-particle states of small lens-shape InAs/GaAs self-assembled quantum dots. The study has been performed in the framework of an empirical tight-binding approximation. We have shown that both the strain and spin-orbit interactions are strong. They influence the number and splitting of the bound states, their density distribution, density space-polarization and transition rates. Although both effects change the WL band edges and the effective energy gap in QD in the opposite way, they are not additive when taken into account simultaneously. The investigated effects cannot be neglected in any model describing properties of InAs/GaAs nanoheterostructures. In contrast, piezoelectric effect is almost negligible in such small dots. The piezoelectric potential change the energies of bound electron and hole states by less than $6 \mathrm{meV}$ and introduce no visible changes on the density distributions.

\section{Acknowledgments}

This work has been supported by the Polish grants 3T1104326 and PZBMIN-008/P03/2003 of the State Committee for Scientific Research. J.A. thanks the Gipuzkoa Forualdundia Fellows Program for financial support.

\section{References}

[1] M. Grundmann, Physica E 5, 167 (1999).

[2] D. Bimberg, M. Grundmann, N.N. Ledentsov, Quantum Dot Heterostructures, Wiley, New York 1999.

[3] M. Bayer, P. Hawrylak, K. Hinzer, S. Fafard, M. Korkusinski, Z.R. Wasilewski, O. Stern, A. Forchell, Science 291, 451 (2001).

[4] G. Bester, J. Shumway, A. Zunger, Phys. Rev. Lett. 93, 047401 (2004).

[5] R. Santoprete, Belita Koiller, R.B. Capaz, P. Kratzer, Q.K.K. Liu, M. Scheffler, Phys. Rev. B 68, 235311 (2003).

[6] S. Lee, F. Oyafuso, P. von Allmen, G. Klimeck, Phys. Rev. B 69, 045316 (2004).

[7] O. Stier, M. Grundmann, D. Bimberg, Phys. Rev. B 59, 5688 (1999).

[8] G. Bester, A. Zunger, Phys. Rev. B 71, 045318 (2005).

[9] Landolt-Börnstein, Numerical Data and Functional Relationships in Science and Technology, Ed. O. Madelung, Springer-Verlag, Berlin 1982.

[10] T.B. Boykin, G. Klimeck, R.C. Bowen, F. Oyafuso, Phys. Rev. B 66, 125207 (2002).

[11] G.W. Bryant, W. Jaskólski, Phys. Rev. B 67, 205320 (2003). 
[12] S. Fafard, M. Spanner, J.P. McCaffrey, Z.R. Wasilewski, Appl. Phys. Lett. 76, 2268 (2000).

[13] T. Hanada, T. Yao, Microel. J., in press.

[14] P. Vogl, H.P. Hjalmarson, J.D. Dow, J. Phys. Chem. Solids 44, 365 (1983).

[15] W. Jaskólski, M. Zieliński, G.W. Bryant, Acta Phys. Pol. A 106, 193 (2004).

[16] S. Lee, O.L. Lazarenkova, P. von Allmen, F. Oyafuso, G. Klimeck, Phys. Rev. B 70, 125307 (2004).

[17] C. Pryor, J. Kim, L.W. Wang, A.J. Williamson, A. Zunger, J. Appl. Phys. 83, 2548 (1998).

[18] T. Saito, Y. Arakawa, Physica E 15, 169 (2002).

[19] W.H. Press, B.P. Flannery, S.A. Teukolsky, W.T. Vetterling, Numerical Recipes, Cambridge University Press, Cambridge 1986.

[20] J.C. Slater, G.F. Koster, Phys. Rev. 94, 1498 (1954).

[21] G. Klimeck, R.C. Bowen, T.B. Boykin, T.A. Cwik, Superlattices Microstruct. 27, 519 (2000).

[22] S. Fraga, J. Muszyńska, Atoms in External Fields, Elsevier, New York 1981.

[23] J.P. Loehr, Physics of Strained Quantum Well Lasers, Kluwer, Boston 1998. 